\title{
Exfoliation of Threading Dislocation-Free, Single-Crystalline, Ultrathin Gallium Nitride Nanomembranes
}

\author{
Rami T. ElAfandy, Mohammed A. Majid, Tien Khee Ng, Lan Zhao, Dongkyu Cha, \\ and Boon S. Ooi\%
}

Despite the recent progress in gallium nitride ( $\mathrm{GaN})$ growth technology, the excessively high threading dislocation (TD) density within the GaN crystal, caused by the reliance on heterogeneous substrates, impedes the development of high-efficiency, low-cost, GaN-based heterostructure devices. For the first time, the chemical exfoliation of completely TD-free, single-crystalline, ultrathin (tens of nanometers) GaN nanomembranes is demonstrated using UV-assisted electroless chemical etching. These nanomembranes can act as seeding layers for subsequent overgrowth of high-quality $\mathrm{GaN}$. A model is proposed, based on scanning and transmission electron microscopy as well as optical measurements to explain the physical processes behind the formation of the GaN nanomembranes. These novel nanomembranes, once transferred to other substrates, present a unique and technologically attractive path towards integrating high-efficiency $\mathrm{GaN}$ optical components along with silicon electronics. Interestingly, due to their nanoscale thickness and macroscopic sizes, these nanomembranes may enable the production of flexible GaN-based optoelectronics.

\section{Introduction}

Gallium nitride (GaN) based light emitting diodes (LEDs) and laser diodes have ushered the way for several staggering technologies including solid-state lighting, high-density optical data storage, high power electronics and laser based projectors and TVs..$^{[1,2]}$ Further scientific advancements and technological breakthroughs are however hindered by the reliance on the existing heterogeneous substrates. Owing to their large thermal expansion and lattice mismatches, the resulting GaN templates grown on sapphire $(\Delta a / a \cong 16 \%)$ substrates suffer from an excessively high threading dislocation (TD) density, on

\footnotetext{
R. T. ElAfandy, Dr. M. A. Majid, Dr. T. K. Ng,

Prof. B. S. Ooi

Photonics Laboratory

Computer, Electrical and Mathematical Sciences

and Engineering

King Abdullah University of Science and Technology (KAUST)

Thuwal, 23955-6900, Kingdom of Saudi Arabia

E-mail: boon.ooi@kaust.edu.sa

Dr. L. Zhao, Dr. D. Cha

Core Lab of Imaging and Characterization

King Abdullah University of Science and Technology (KAUST)

Thuwal, 23955-6900, Kingdom of Saudi Arabia

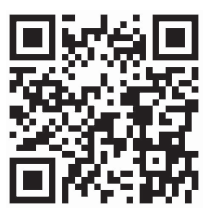

DOI: 10.1002/adfm.201303001 the order of $10^{10} \mathrm{~cm}^{-2}$, causing a decrease in the material quantum efficiency and a reduction in the device lifetime. ${ }^{[3]}$ Several techniques were implemented to lower the TD density within the epitaxial GaN crystal including; epitaxial lateral overgrowth (ELOG), ${ }^{[4]}$ dislocation filtering, ${ }^{[5]}$ growth of aluminum nitride (AlN) or GaN buffer layers, ${ }^{[6,7]}$ or overgrowth on $\mathrm{GaN}$ nanocolumns. ${ }^{[8]}$ Other fabrication technologies do not depend on epitaxial growth, such as ammonothermal growth, which successfully produced $\mathrm{GaN}$ with TD density as low as $10^{4} \mathrm{~cm}^{-2}$, yet, the process is relatively costly, energy hungry and suffers from very slow growth rate which make its commercialization challenging. ${ }^{[9,10]}$ Other significant researches include fabricating devices based on GaN nanowires ${ }^{[11]}$ or nano-pyramids ${ }^{[12]}$ grown onto different substrates since these nanostructures are characterized by a lower TD density. There are undoubtedly great economic and technologic interests to produce a TD free GaN 2D layer with the potential of epitaxially overgrowing high quality GaN films in a high throughput, cost effective and energy efficient manner.

Fabrication of free standing nanomembranes has previously been attempted by several groups, ${ }^{[13-18]}$ yet, there are no reports regarding the fabrication of TD free GaN nanomembranes. Here, we demonstrate for the first time the chemical exfoliation of a TD free ultrathin $(20-30 \mathrm{~nm})$, freely suspended, unstrained single crystalline GaN nanomembranes, produced by a cost effective and energy efficient facile technique, UV-assisted electroless etching, with subsequent mechanical transfer to a foreign substrate. ${ }^{[19]}$ The exfoliated TD free unstrained nanomembranes, once transferred, can potentially act as a seeding layer for the epitaxial overgrowth of high quality GaN. Moreover, due to their nano-scale thickness and macroscopic sizes, these nanomembranes present a possible novel gateway for the production of high efficiency flexible GaN optoelectronic devices. A model, based on the growth kinetics combined with the electroless etching carrier dynamics, is developed to provide a plausible explanation for the formation of these novel nanomembranes. Results based on the micro-photoluminescence ( $\mu \mathrm{PL})$, transmission electron microscopy (TEM) and energy dispersive X-ray spectroscopy (EDS) support the proposed model and demonstrate the single crystalline nature of the prepared nanomembranes. 


\section{Results and Discussion}

\subsection{Exfoliation Model}

The TD free GaN nanomembranes are exfoliated by means of UV-assisted electroless etching of $\mathrm{n}-\mathrm{GaN}$ in $\mathrm{HF}$ based electrolyte $\left(\mathrm{CH}_{3} \mathrm{OH}: \mathrm{H}_{2} \mathrm{O}_{2}: \mathrm{HF}\right.$ in our case). The process depends on the presence of a surface charge region (SCR) at the $n-\mathrm{GaN} /$ electrolyte interface which arises due to GaN surface Fermilevel $\left(E_{\mathrm{f}}\right)$ equilibrium with the electrochemical potential $(\tilde{\mu})$ of the electrolyte (Figure 1a). ${ }^{[20]}$ This SCR is characterized by the presence of surface electrical fields which cause an upward bending in the energy bands near the surface. UV-assisted electroless etching of $\mathrm{n}-\mathrm{GaN}$ initiates when incident UV photons excite electron-hole $\left(\mathrm{e}^{-}, \mathrm{h}^{+}\right)$pairs. If the carriers are excited up to the hole diffusion length $\left(\mathrm{L}_{\mathrm{H}}\right)$ away from the SCR, holes may diffuse to the SCR where they drift towards the interface under the effect of the surface electric fields, else, they simply recombine. Once the photo-generated holes reach the GaN/electrolyte interface, they get localized at the surface bonds, allowing the surface gallium atoms to get oxidized forming gallium oxide $\left(\mathrm{Ga}_{2} \mathrm{O}_{3}\right)$ according to the following oxidation reaction: ${ }^{[21,22]}$

$$
\begin{aligned}
& 2 \mathrm{GaN}(\mathrm{s})+6 \mathrm{~h}^{+}+3 \mathrm{H}_{2} \mathrm{O}(1) \rightarrow \mathrm{Ga}_{2} \mathrm{O}_{3}(\mathrm{~s}) \\
& +6 \mathrm{H}^{+}(\mathrm{ag})+\mathrm{N}_{\text {○ }}(\mathrm{g})
\end{aligned}
$$

a

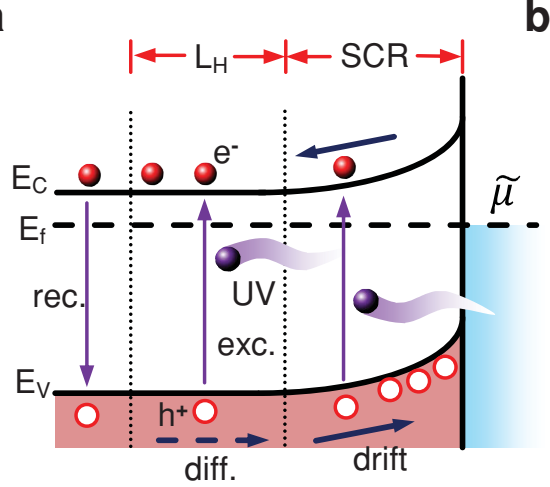

C

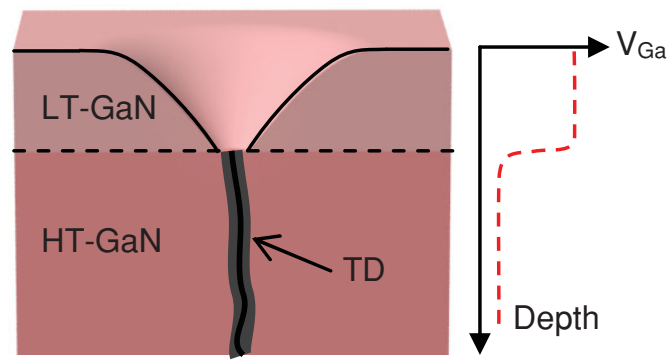

Figure 1. The energy band diagrams of the $\mathrm{GaN}$ in equilibrium with the electrolyte, in the case of high doping concentration (a) or the case of doping compensation (b), showing the surface energy band bending in each case. (c) Schematic view of $\mathrm{GaN}$ substrate grown under high temperature $(\mathrm{HT}-\mathrm{GaN})\left(\sim 1050^{\circ} \mathrm{C}\right)$ followed by $\mathrm{GaN}$ grown under low temperature (LT-GaN) $\left(\sim 700^{\circ} \mathrm{C}\right)$ causing the formation of a layer void of dislocation and containing an increased density of gallium vacancies $\left(\mathrm{V}_{\mathrm{Ca}}\right)$. The curve on the right depicts the decrease in the $\mathrm{V}_{\mathrm{Ga}}$ density from the LT-GaN to the HT-GaN.
After oxidation, $\mathrm{Ga}_{2} \mathrm{O}_{3}$ is then etched away by $\mathrm{HF}$. Decreasing the surface energy band bending, whether by reducing $\mathrm{n}$-doping (silicon) or increasing compensation by p-doping causes the hole drift to the surface, and hence the etching process, to slow down (Figure 1b). One possible way of compensating the Si dopants is by increasing the density of gallium vacancy defects $\left(\mathrm{V}_{\mathrm{Ga}}\right)$ which behave as deep acceptors. ${ }^{[23]}$ Regions of higher $\mathrm{V}_{\mathrm{Ga}}$ densities will be more resistant to UVassisted electroless etching due to the decreased energy band bending. This concept was previously employed to create $\mathrm{GaN}$ nanomembranes by creating surface $\mathrm{V}_{\mathrm{Ga}}$ through argon bombardment. ${ }^{[24]}$ The novel process presented here, to form TD free $\mathrm{GaN}$ nanomembranes, is believed to originate from the varying $\mathrm{V}_{\mathrm{G} a}$ density depth profile due to varying temperature during growth. Hydride vapor phase epitaxy (HVPE) growth of GaN at high temperatures (HT-GaN) $\left(1050-1075{ }^{\circ} \mathrm{C}\right)$ typically results in a high quality $\mathrm{GaN}$ crystal, with low $\mathrm{V}_{\mathrm{G} a}$ density, since $\mathrm{Ga}$ adatoms acquire enough thermal energy to overcome the lattice potential barrier and migrate till they get incorporated in low energy lattice sites. ${ }^{[25]}$ However, GaN growth at lower temperatures (LT-GaN) $\left(700-750^{\circ} \mathrm{C}\right.$ ) causes the process to shift from being thermodynamically controlled to kinetically controlled and hence shortening the adatoms diffusion length, rendering them unable to reach the low energy lattice sites. ${ }^{[26]}$ Thus the $\mathrm{V}_{\mathrm{Ga}}$ density increases and the material becomes less n-type, due to the dopant compensation. Interestingly, concurrent to the increase in the $\mathrm{V}_{\mathrm{Ga}}$ density, at low temperatures, there is a reduction in $\mathrm{Ga}$ incorporation on the $\{10 \overline{1} 1\}$ planes in comparison with the (0001) surface causing the $\mathrm{Ga}$ adatoms to get incorporated away from the TD terminations on the (0001) surface. ${ }^{[27,28]}$ Thus, it can be inferred that the growth of tens of nanometers of LT-GaN on top of HT-GaN will form a layer void of TDs and contains an increased $\mathrm{V}_{\mathrm{Ga}}$ density (Figure 1c). This proposed model will be utilized in this work to explain the formation of the TD free GaN nanomembranes which, to the best of our knowledge, are observed for the first time.

\subsection{Exfoliation Process}

The exfoliation process of the TD free nanomembrane is believed to originate from unintended growth temperature fluctuations at the last decades of nanometers that resulted in an increased $\mathrm{V}_{\mathrm{Ga}}$ density. The process is illustrated, step by step, by means of the SEM images presented in Figure 2 . Since the surface contains a higher $\mathrm{V}_{\mathrm{Ga}}$ density the surface energy bands are just slightly bent causing a slower etching rate than the bulk. The regions containing low $\mathrm{V}_{\mathrm{Ga}}(\mathrm{HT}-\mathrm{GaN})$, and hence are etched at a much higher rate, are only in contact with the electrolyte at sites where TD terminate on the surface (Figure 1c) and thus this is where the surface etch pores nucleate (Figure 2a). The pores then proceed along the [0001] crystallographic direction (yellow arrow in Figure 2b). Simultaneously with the vertical etching, lateral etching proceeds $^{[29,30]}$ (red arrows in Figure 2c) underneath the LT-GaN causing the local exfoliation of the top surface. As the lateral etching proceeds, secondary vertical pores nucleate and propagate crystallographically along the [0001] direction (Figure 2c). The presence of these two etching mechanisms causes the 

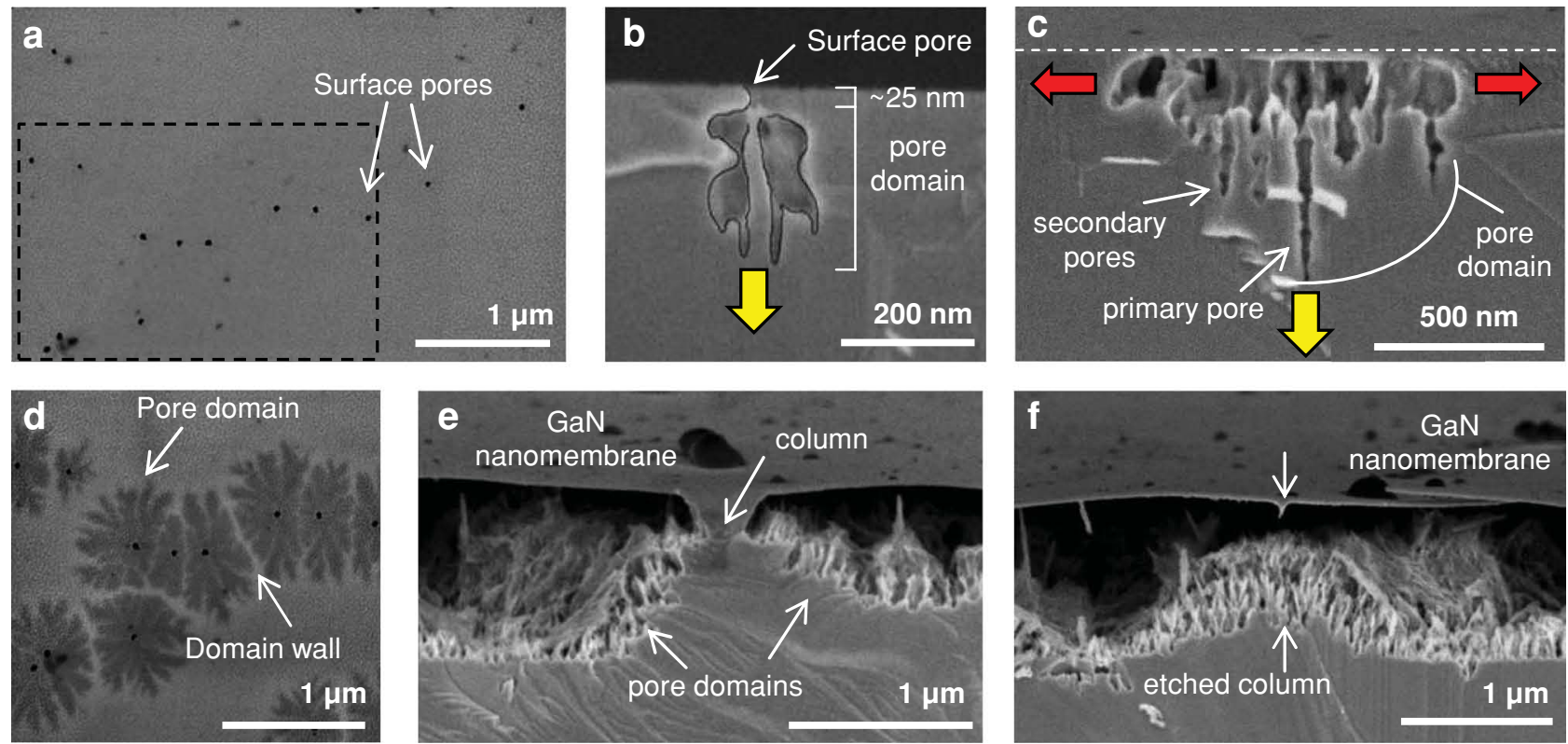

Figure 2. SEM micrographs showing different stages along the nanomembrane formation. (a) Top view SEM image of the nucleated surface etch pores. (b) Cross sectional SEM micrograph of a nascent pore domain. A nanowire is observed in the middle of the pore domain. The boundary of the domain is marked in black. (c) Inclined side view of a relatively older pore domain encompassing porous GaN formed of a primary and secondary pores. The yellow and red arrows represent the vertical and the lateral etch processes, respectively. The region above the white dashed line is the (0001) surface. (d) Top view SEM image of the region marked with a dashed contour in (a) but using higher acceleration voltage. The embedded pore domains, formed around the surface etch pores, are visible. e, Inclined side view of the nanomembrane is supported by few columns after the domains have coalesced. (e) The nanomembrane lying on a porous GaN layer on top of the bulk GaN.

formation of undersurface cavity like structures, or pore domains, ${ }^{[31,32]}$ which encompass a group of spatially confined vertical pores with a tiny opening at the top. Top view, high $\mathrm{kV}$ SEM image in Figure 2d reveals the pore domains spreading laterally underneath the surface layer. Since the pore domains are only embedded tens nanometers deep from the surface, a considerable amount of UV photons can reach them generating e-h pairs on the domain walls allowing the UV-assisted etching of the HT-GaN to proceed. As observed in Figure 2d, etching slows down as the pore domains start to approach each other due to the surface energy band flattening in the SCR causing the formation of domain walls. However, at a later time, even the domain walls get etched away allowing the coalescence of the pore domains. Later, the surface layer is only supported by few columns which also eventually get etched forming a freely suspended 20-30 nm thin GaN nanomembrane on top of a porous GaN layer (Figure 2e,f). The sample is then dried using a critical point drier (CPD) with liquid carbon dioxide to prevent the nanomembrane from collapsing downward and sticking to the porous GaN. Since the surface energy bands of the LT-GaN still are slightly bended (Figure 1b), if the process is left to proceed, the nanomembrane itself will get etched away. Any lateral variations in the $V_{\mathrm{Ga}}$ density within the LT-GaN, due to some local differences in the growth conditions, cause some regions within the nanomembrane to become more resistant to etching than others. So, while the process is potentially able to exfoliate nanomembranes of different areas (up to centimeters squared), some regions (of low $\mathrm{V}_{\mathrm{Ga}}$ density) get etched away before other regions (of high $\mathrm{V}_{\mathrm{Ga}}$ density) get completely exfoliated, and thus adding a limitation on the lateral dimensions of the nanomembranes. This issue could be resolved by having a more controlled uniform growth of the LT-GaN across the wafer as well as lowering the growth temperature enough to completely flatten the energy bands within the LT-GaN. Based on the proposed model, the nanomembranes' thickness is exactly that of the LT-GaN and so, varying the thickness of the LT-GaN can be used to engineer the nanomembrane thickness. However, there has to be an upper limit, which is clearly a function of growth conditions, where the thick LT-GaN will cover the TD termination, ${ }^{[27]}$ blocking the electrolyte pathway to the HT-GaN and thus preventing etching from initiation.

Nanowires (NWs), or whiskers, are common to form during UV-assisted electroless etching from TDs which offer fast recombination channels for the photo-generated holes, and thus depleting the surrounding holes preventing etching of the neighboring region. ${ }^{33]}$ Through careful examination of the SEM images (Figure 3a), the tips of the NWs are observed directly below the surface etch pits which validates our process for exfoliating TD free GaN nanomembranes. Upon drying (without using a CPD), the nanomembrane collapses downward under the effect of the water surface tension causing the NWs to protrude through it via the surface etch pores (Figure 3b,e). The cross sectional TEM images of the surface pore domains reveals TDs running through their centers as previously predicted from the proposed model (the TEM image of a typical pore domain is presented in Figure 3f). Figure 3c clearly shows the cross sectional TEM image of the GaN nanomembrane. The 3D illustration, presented in Figure 3d, depicts a typical 

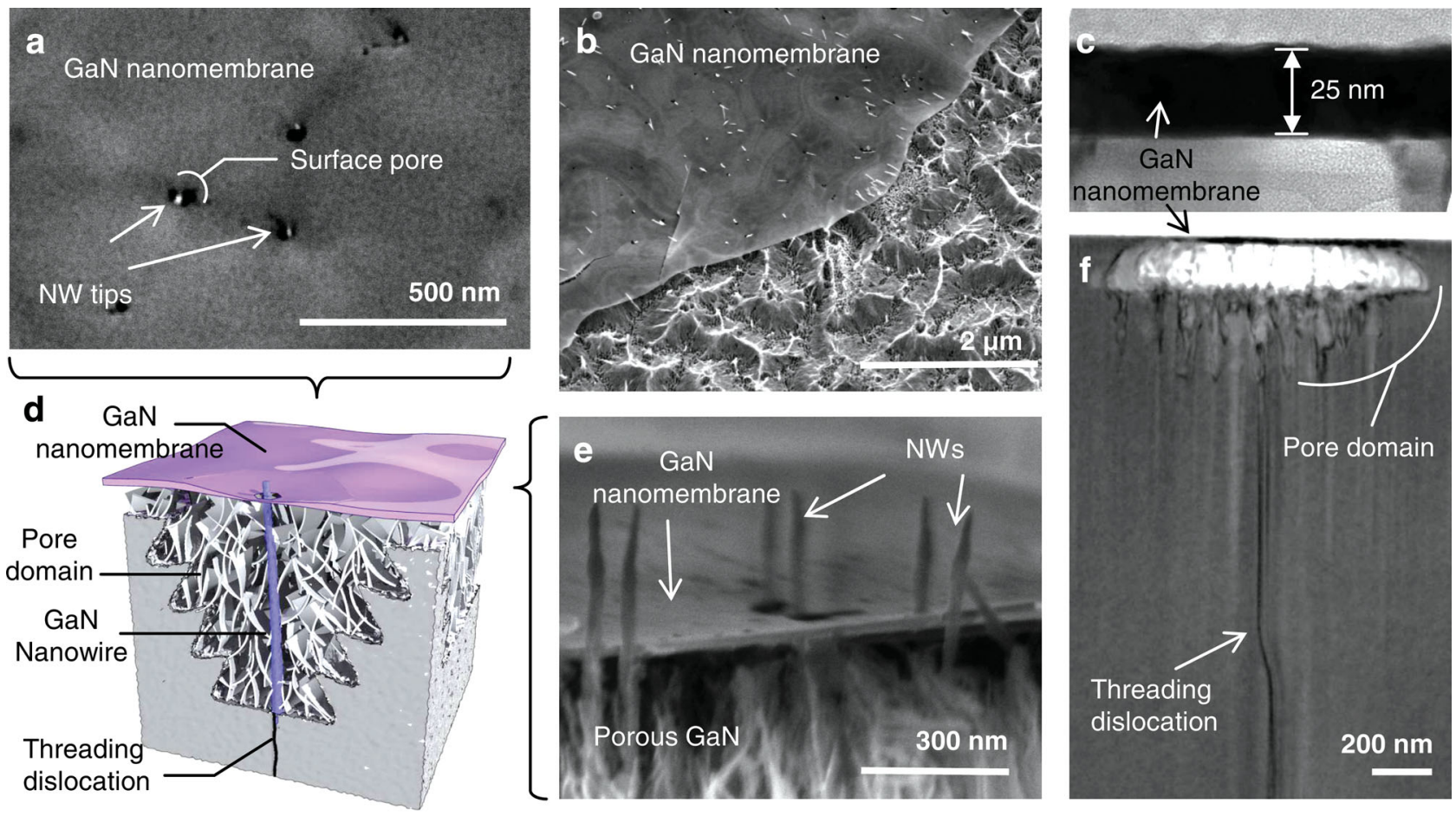

Figure 3. (a) SEM image showing the tips of the NWs below the surface pores. (b) Inclined top view SEM image of the nanomembrane on top of the porous GaN. (c) Cross sectional TEM image of the $25 \mathrm{~nm}$ thin nanomembrane. (d) 3D illustration of a typical pore domain with the GaN TD free nanomembrane on top of it. (e) inclined cross sectional SEM image of NWs protruding through the surface pores in the GaN nanomembrane. $(f)$ Cross sectional TEM image of a pore domain formed exactly around a TD with the nanomembrane on top of it.

pore domain encompassing porous GaN with one NW, formed from a TD, running through its center and protruding through the top $\mathrm{GaN}$ nanomembrane via the surface etch pore.

\subsection{Crystal Structure Analysis}

The crystalline nature of the exfoliated nanomembrane is critical for achieving a high quality overgrown GaN since an amorphous structure will induce lattice dislocations during growth causing the production of low quality GaN. Thus, a small piece of the GaN nanomembrane is mechanically transferred, by means of a $40 \mu \mathrm{m}$ tungsten probe (Figure $4 \mathrm{a}$ ) to a carbon coated copper grid (Figure 4b) for TEM imaging. The high resolution TEM (HRTEM) of the nanomembrane, with imaging axis aligned to the c-plane, is presented in Figure $\mathrm{c}$ and the produced electron diffraction (ED) pattern shown in Figure 4d. The high degree of crystallinity of the hexagonal crystal lattice and its diffraction pattern are clearly observed. Measuring the inter-planar distances from the (1010), (0110), and ( $\overline{1} 100)$ diffraction spots yields a value of $2.738 \AA$ which is close to the tabulated data for the unstrained GaN. These measurements conclusively indicate that the process described above successfully produces TD free, single crystalline GaN nanomembranes. To the best of our knowledge, no other group has reported the exfoliation of $\mathrm{GaN}$ nanomembranes with these properties before. The collected energy dispersive X-ray spectroscopy (EDS) spectrum from the GaN nanomembrane in Figure 4e reveals no peaks other than that associated with $\mathrm{N}$, fluorine $(\mathrm{F})$ and $\mathrm{Ga}$. The F peak is believed to originate from residual $\mathrm{F}$ halogens that tie up with the Ga dangling bonds at the surface which is usually the case after nitrides exposure to HF. ${ }^{[34]}$

Prior to complete exfoliation, the SEM image in Figure $4 \mathrm{f}$ shows that the NW, formed from a TD, is in direct contact with the etch pore present in the nanomembrane. Since, during the overgrowth process, any remnants of the dislocated crystal sites in the nanomembrane will nucleate further TDs within the overgrown crystal, we next demonstrate that the TDs' cores are completely detached from the nanomembrane during the exfoliation process. After total exfoliation, the etch pores within the nanomembrane are imaged using TEM (a typical pore is presented in Figure 4g). Instead of having a hexagonal boundary, the etch pore has more of a heart shaped boundary, for yet unclear reasons, where the center, marked with a circle, was in direct contact with the NW tip. In Figure 4h, an HRTEM image of the center region of the etch pore, clearly shows a single crystalline lattice, leaving no doubt that the entire nanomembrane is TD free.

\subsection{Optical Characterization}

After demonstrating the crystalline nature of the TD free GaN nanomembranes, we further characterize their optical properties using micro-photoluminescence $(\mu \mathrm{PL})$ and Raman spectroscopy. In order to remove any interference from the much 

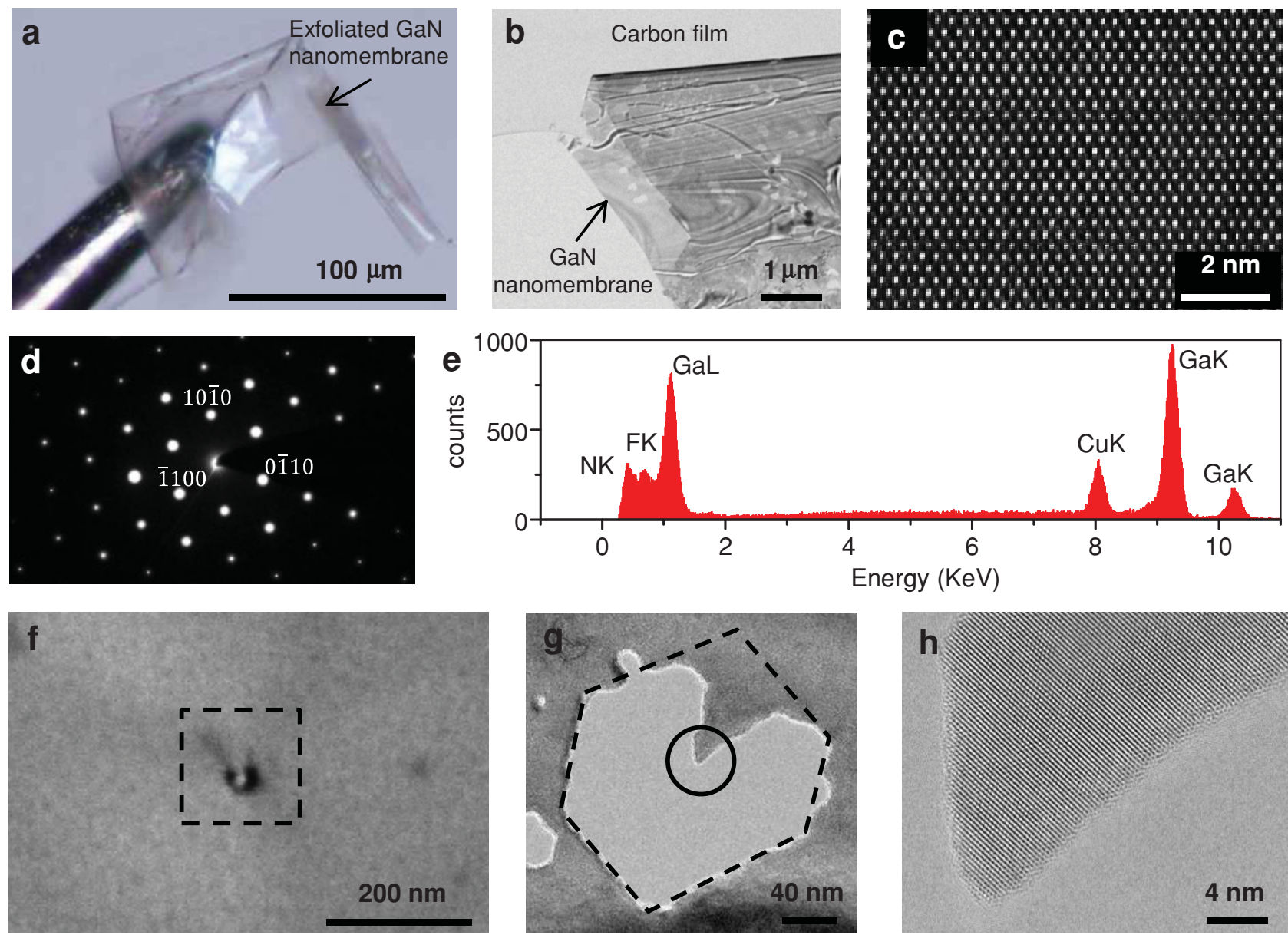

Figure 4. (a) Optical image of an exfoliated nanomembrane while being attached to a $40 \mu \mathrm{m}$ wide tungesten probe. (b) TEM image of a GaN nanomembrane after transfer onto a carbon coated cupper TEM grid. (c) HRTEM of the nanomembrane pictured along the [0001] zone axis showing the perfect hexagonal crystalline structure of the nanomembrane. (d) The electron diffraction pattern recorded along the [0001] zone axis with the marked (1010), $(0 \overline{1} 10)$ and ( $\overline{1} 100)$ diffraction spots. (e) The collected EDS spectrum from the GaN nanomembrane attached to the copper grid. (f) SEM image of one surface pore in a nanomembrane with the underlying NW in direct contact with it. (g) TEM image of a heart shaped surface pore in the nanomembrane after complete detachment from the TD within the underlying porous layer. (h) HRTEM micrograph of the center of the etch pit (marked with a circle in (g)) clearly showing a single crystal with no presence of a crystalline dislocation.

stronger $\mu \mathrm{PL}$ signal of the underlying porous and bulk GaN, the nanomembrane is transferred to a sapphire substrate $\left(\mathrm{E}_{\mathrm{g}}=9.9 \mathrm{eV}\right)$ as shown in Figure 5a inset (i) and schematically depicted in inset (ii). In Figure 5a we plot the normalized $\mu \mathrm{PL}$ signals measured from a GaN wafer and from a transferred GaN nanomembrane under the same excitation conditions. While both the nanomembrane and the GaN wafer exhibit $\mu \mathrm{PL}$ peak emissions at approximately $3.4 \mathrm{eV}$ which is the characteristic band to band transition in GaN, only the $\mu \mathrm{PL}$ emission from the GaN nanomembrane demonstrates a broad emission between 2.1 and $2.4 \mathrm{eV}$ (yellow luminescence (YL)) which is attributed to the conduction band to the $\mathrm{V}_{\mathrm{Ga}}$ impurity level transition. ${ }^{[35]}$ This unambiguously demonstrates the more pronounced $\mathrm{V}_{\mathrm{Ga}}$ density in the nanomembrane as compared to that of the $\mathrm{GaN}$ wafer which is in agreement with the model presented earlier in this work.

Since overgrowth on lattice miss-matched substrates leads to the nucleation of strain-relief defects such as in-plane dislocations, TDs or stacking faults which are detrimental to the overall device efficiency, it is imperative to analyze the internal strain within the nanomembrane. The first order Raman signal is collected under the $\mathrm{z}(\mathrm{x}, \mathrm{x}+\mathrm{y}) \overline{\mathrm{z}}$ scattering configuration causing $\mathrm{E}_{2}^{\mathrm{H}}, \mathrm{E}_{2}^{\mathrm{L}}$, the high and low frequency branches of $E_{2}$, respectively, and $A_{1}(L O)$ to become the only allowed optical phonon modes during the measurements (Figure $5 \mathrm{~b}$ ). Since the nanomembrane is only $25 \mathrm{~nm}$ thick, the measured signal to noise ratio is relatively low causing the $A_{1}(L O)$ and the $\mathrm{E}_{2}^{\mathrm{L}}$ phonon signal to become completely masked (refer to Supporting Information). Since the atomic vibrations within the $E_{2}^{H}$ phonon mode are lateral (perpendicular to the c-axis), red arrows in Figure 5b, this mode is highly sensitive to the biaxial strain within the GaN crystal. ${ }^{[36]}$ The black curve in Figure 5b is the measured Raman shift from the $\mathrm{E}_{2}^{\mathrm{H}}$ phonon mode of a GaN wafer prior to any chemical contamination. The peak signal $\left(570 \mathrm{~cm}^{-1}\right)$ is clearly shifted to higher vibrational frequencies from the zero strain line $\left(568 \mathrm{~cm}^{-1}\right)^{[37]}$ basically due to the residual compressive strain from the sapphire substrate. On the other hand, the Raman peak signal at $567.7 \mathrm{~cm}^{-1}$ belongs 

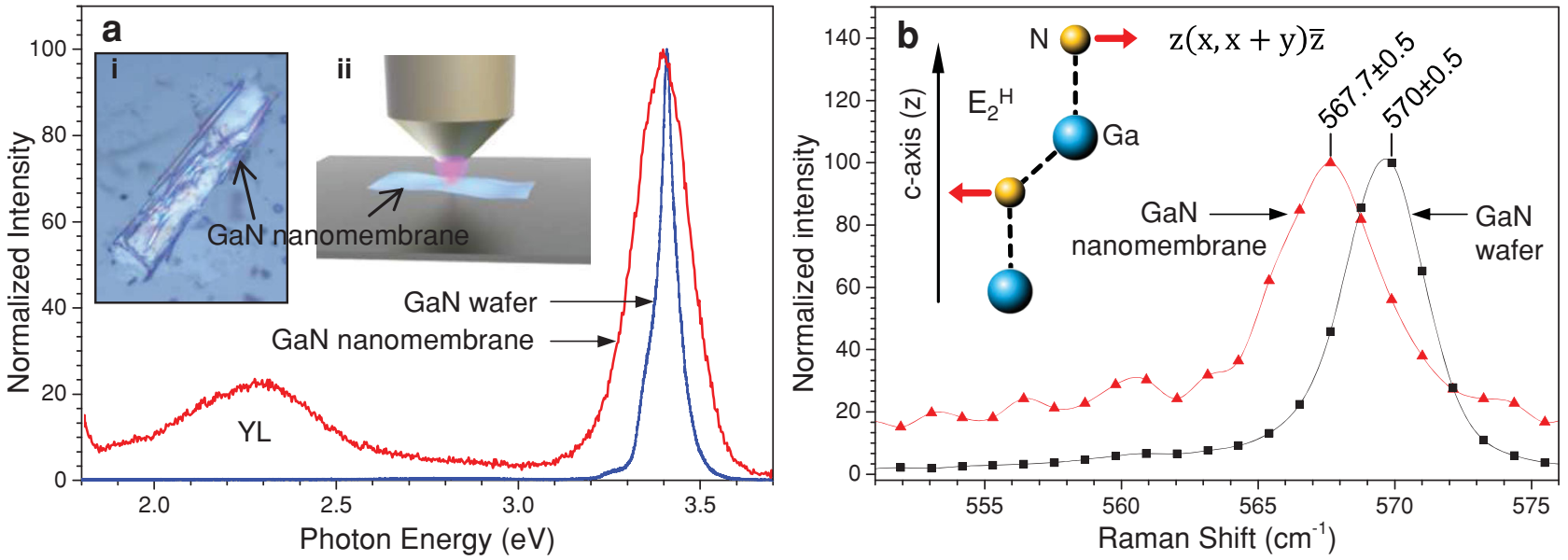

Figure 5. Optical measurements onto the nanomembrane. (a) The recorded $\mu \mathrm{PL}$ peak emission signal from the GaN nanomembrane and a GaN wafer. While both of them peak at approximately $3.4 \mathrm{eV}$, only the $\mu \mathrm{PL}$ signal from the GaN nanomembrane shows an emission at the low energy region (2.1-2.4 eV). Inset (i) shows the optical image of the transferred GaN nanomembrane onto the sapphire substrate. Inset (ii) shows a 3D illustration of the GaN TD free nanomembrane onto sapphire while being probed by a UV laser focused by an objective lens. (b) First order Raman signal measured from a GaN wafer, with sapphire substrate, (black squares) and from the exfoliated nanomembrane (red triangles) show different Raman shifts. The vibration of the $E_{2}^{H}$ phonon mode is depicted as consecutive $N$ atoms vibrating with a $\pi$ phase difference.

to the nanomembrane after exfoliation from the GaN wafer. It is observed that the originally compressively strained crystal of the nanomembrane gets completely relaxed during the exfoliation process which further reduces the probability of nucleating strain-relief defects during epitaxial overgrowth.

\section{Conclusion}

We have successfully demonstrated, for the first time, the exfoliation of a TD free, freely suspended, single crystalline unstrained GaN nanomembrane through a facile, inexpensive and energy efficient technique; UV-assisted electroless etching, from a HVPE grown GaN wafer having a $10^{8} \mathrm{~cm}^{-2}$ TD density. An unintended growth temperature fluctuation is believed to have occurred which rendered the top surface of the GaN wafer, except regions of TD termination, more resistant towards the etching process. We proposed a model based on the previous speculation to explain the formation process of the TD free nanomembranes. Presented SEM and TEM images as well as EDS and $\mu \mathrm{PL}$ measurements support the proposed model and demonstrate the complete exfoliation procedure of the nanomembranes. Raman measurements were performed to reveal the fully relaxed $\mathrm{GaN}$ crystal within the nanomembrane. The process can be further engineered in terms of varying the growth conditions of the LT-GaN (thickness, temperature and doping concentration) in order to exfoliate nanomembranes of different thicknesses and areas. Moreover, chemical compositions, GaN surface preparation and radiation intensities can be optimized to control the exfoliate process. These TD free nanomembranes offer a unique potential and a technologically attractive path for overgrowth of high quality GaN based optical devices.

\section{Experimental Section}

Preparation of the GaN Samples: The GaN wafers used in this study consisted of $30 \mu \mathrm{m}$ of silicon doped $\left(\sim 10^{18} \mathrm{~cm}^{-3}\right)$ c-plane oriented GaN grown using hydride vapor phase epitaxy (HVPE) technique on a sapphire substrate. The wafers have a TD density of $10^{8} \mathrm{~cm}^{-2}$. There is an ambiguity in the top 20-30 nm where we believe that a decrease in growth temperature has occurred. The $\mathrm{GaN}$ wafers were cleaved into $1 \mathrm{~cm}^{2}$ pieces which were later degreased in acetone and isopropanol alcohol (IPA), respectively for 5 mins and finally cleaned in hot $\mathrm{HNO}_{3}$ $\left(65^{\circ} \mathrm{C}\right.$ ) for $15 \mathrm{~min}$ for surface oxide removal. Some other samples were further cleaned into $\mathrm{HCL}$ for $10 \mathrm{~min}$ or in $\mathrm{HF}$ for $2 \mathrm{~h}$ to be certain of a complete surface oxide removal. ${ }^{[38]}$ After covering most of the sample surfaces by a glass slide, a thin layer of platinum metal $(150 \mathrm{~nm})$ was deposited using a reactive ion sputter $R$ and $D$ system from equipment support company (ESC).

Preparation of the Free-Standing GaN Nanomembranes: The GaN samples were placed in a bath containing $\mathrm{CH}_{3} \mathrm{OH}: \mathrm{H}_{2} \mathrm{O}_{2}(35 \%): \mathrm{HF}(48 \%)$ (1:2:2) with the c-plane in contact with the solution. A fused silica lens was used to focus ultraviolet (UV) light emanating from a $200 \mathrm{~W}$ mercury $(\mathrm{Hg})$ arc lamp onto the sample surface uncovered with platinum. Once the desired etching period was reached, the samples were cleaned by dipping and rinsing in IPA and then dried using a critical point dryer (CPD).

Characterization of the Dislocation Free GaN Nanomembranes: SEM and TEM images were recorded using the Nova NanoSEM and the Titan G2 60-300 TEM from FEI, respectively. Surface topography was recorded using the 5400 AFM from Agilent. The GaN nanomembranes were transferred to other materials (sapphire or carbon coated cupper grid) by lifting them with a $40 \mathrm{~nm}$ thick tungsten probe and later depositing them elsewhere. To measure the $\mu \mathrm{PL}$ signal from the nanomembrane, a $325 \mathrm{~nm}$ laser $\left(\mathrm{E}_{\mathrm{exc}}=3.8 \mathrm{eV}\right)$ from a He-Cd gas laser was focused to a spot size of $28 \mu \mathrm{m}^{2}$. To measure Raman signal, the nanomembrane was transferred to a carbon coated TEM copper grid and a $473 \mathrm{~nm}$ laser was focused by a 100x objective lens onto the holes within the carbon film in order to remove any interference from the underlying material. The focused laser beam as well as the collected scattered beam did not pass through any polarizers. 


\section{Supporting Information}

Supporting Information is available from the Wiley Online Library or from the author.

\section{Acknowledgements}

Rami T. ElAfandy designed and performed the experiments, interpreted the data and wrote the manuscript. Mohammed A. Majid, Tien Khee Ng, Boon S. Ooi helped in writing the manuscript. Lan Zhao and Dongkyu Cha performed the transmission electron microscopy. Boon S. Ooi supervised the project. All authors approved the final manuscript.

Received: August 28, 2013 Revised: October 23, 2013 Published online:

[1] F. Ponce, D. Bour, Nature 1997, 386, 351.

[2] S. F. Chichibu, A. Uedono, T. Onuma, B. A. Haskell, A. Chakraborty, T. Koyama, P. T. Fini, S. Keller, S. P. DenBaars, J. S. Speck, Nature Mater. 2006, 5, 810.

[3] M. A. Moram, R. A. Oliver, M. J. Kappers, C. J. Humphreys, Adv. Mater. 2009, 21, 3941.

[4] O. H. Nam, M. D. Bremser, T. S. Zheleva, R. F. Davis, Appl. Phys. Lett. 1997, 71, 2638.

[5] R. Colby, Z. Liang, I. H. Wildeson, D. A. Ewoldt, T. D. Sands, R. E. García, E. A. Stach, Nano Lett. 2010, 10, 1568.

[6] S. Yoshida, S. Misawa, S. Gonda, Appl. Phys. Lett. 1983, 42, 427.

[7] J. Bai, T. Wang, P. Comming, P. Parbrook, J. David, A. Cullis, J. Appl. Phys. 2006, 99, 023513.

[8] T. Y. Tang, W. Y. Shiao, C. H. Lin, K. C. Shen, J. J. Huang, S. Y. Ting, T. C. Liu, C. Yang, C. L. Yao, J. H. Yeh, J. Appl. Phys. 2009, 105, 023501.

[9] T. Hashimoto, K. Fujito, F. Wu, B. A. Haskell, P. T. Fini, J. S. Speck, S. Nakamura, Jap. J. Appl. Phys. 2005, 44, L797.

[10] T. Hashimoto, F. Wu, J. S. Speck, S. Nakamura, Nature Mater. 2007, $6,568$.

[11] W. Guo, M. Zhang, A. Banerjee, P. Bhattacharya, Nano Lett. 2010, 10, 3355.

[12] J. H. Choi, A. Zoulkarneev, S. I. Kim, C. W. Baik, M. H. Yang, S. S. Park, H. Suh, U. J. Kim, H. B. Son, J. S. Lee, Nature Phot. 2011, $5,76$.

[13] S. Balendhran, S. Walia, H. Nili, J. Z. Ou, S. Zhuiykov, R. B. Kaner, S. Sriram, M. Bhaskaran, K. Kalantar-zadeh, Adv. Funct. Mater. 2013, 23, 395.

[14] S. H. Chae, W. J. Yu, J. J. Bae, D. L. Duong, D. Perello, H. Y. Jeong, Q. H. Ta, T. H. Ly, Q. A. Vu, M. Yun, Nature Mater. 2013, 12, 40.
[15] J. N. Coleman, M. Lotya, A. O'Neill, S. D. Bergin, P. J. King, U. Khan, K. Young, A. Gaucher, S. De, R. J. Smith, Science 2011, 331, 56.

[16] F. Cavallo, M. G. Lagally, Soft Matter 2009, 6, 425.

[17] Y. Mei, D. J. Thurmer, C. Deneke, S. Kiravittaya, Y.-F. Chen, A. Dadgar, F. Bertram, B. Bastek, A. Krost, J. Christen, T. Reindl, M. Stoffel, E. Coric, O. G. Schmidt, ACS Nano 2009, 3, 1663.

[18] G. Huang, Y. Mei, Adv. Mater. 2012, 24, 2517.

[19] J. A. Rogers, M. G. Lagally, R. G. Nuzzo, Nature 2011,477, 45.

[20] K. Rajeshwar, in Encyclopedia of Electrochemistry, Vol. 6 (Eds: A. J. Bard, M. Stratmann, S. Licht), Wiley-VCH, Germany 2002, Ch. 1.

[21] C. Youtsey, G. Bulman, I. Adesida, J. Elec. Mater. 1998, 27, 282.

[22] A. Vajpeyi, S. Tripathy, S. Chua, E. Fitzgerald, Phys. E: Low-dimens. Syst. Nanos. 2005, 28, 141.

[23] J. Neugebauer, C. G. V. d. Walle, Appl. Phys. Lett. 1996, 69, 503.

[24] I. Tiginyanu, V. Popa, M. A. Stevens-Kalceff, Mater. Lett. 2011, 65, 360.

[25] K. Oura, V. G. Lifshits, A. A. Saranin, A. V. Zotov, M. Katayama, Surface Science: An Introduction, Springer-Verlag, Berlin Heidelberg, 2003.

[26] H. Morkoç, in Handbook of Nitride Semiconductors and Devices, Vol. 1, Wiley-VCH, Germany 2009, Ch. 4.

[27] X. H. Wu, C. R. Elsass, A. Abare, M. Mack, S. Keller, P. M. Petroff, S. P. DenBaars, J. S. Speck, S. J. Rosner, Appl. Phys. Lett. 1998, 72, 692.

[28] S. Mahanty, M. Hao, T. Sugahara, R. S. Q. Fareed, Y. Morishima, Y. Naoi, T. Wang, S. Sakai, Mater. Lett. 1999, 41, 67.

[29] H. Föll, S. Langa, J. Carstensen, M. Christophersen, I. M. Tiginyanu, Adv. Mater. 2003, 15, 183.

[30] T. L. Williamson, D. J. Diaz, P. W. Bohn, in Porous Silicon Carbide and Gallium Nitride: Epitaxy, Catalysis, and Biotechnology Applications (Eds: R. M. Feenstra, C. E. Wood), Wiley, Chichester, UK 2008, Ch. 4.

[31] B. Erne, D. Vanmaekelbergh, J. Kelly, J. Electrochem. Soc. 1996, 143, 305.

[32] C. O'Dwyer, D. Buckley, D. Sutton, M. Serantoni, S. Newcomb, J. Electrochem. Soc. 2007, 154, H78.

[33] C. Youtsey, L. Romano, I. Adesida, Appl. Phys. Lett. 1998, 73, 797.

[34] S. W. King, J. P. Barnak, M. D. Bremser, K. M. Tracy, C. Ronning, R. F. Davis, R. J. Nemanich, J. Appl. Phys. 1998, 84, 5248.

[35] K. Saarinen, T. Laine, S. Kuisma, J. Nissilä, P. Hautojärvi, L. Dobrzynski, J. M. Baranowski, K. Pakula, R. Stepniewski, M. Wojdak, A. Wysmolek, T. Suski, M. Leszczynski, I. Grzegory, S. Porowski, Phys. Rev. Lett. 1997, 79, 3030.

[36] T. Kozawa, T. Kachi, H. Kano, H. Nagase, N. Koide, K. Manabe, J. Appl. Phys. 1995, 77, 4389.

[37] P. Perlin, C. Jauberthie-Carillon, J. P. Itie, A. San Miguel, I. Grzegory, A. Polian, Phys. Rev. B 1992, 45, 83.

[38] S. Ohira, N. Arai, Phys. Stat. Sol. (c) 2008, 5, 3116. 